\title{
Gauge-invariant signatures of spontaneous gauge symmetry breaking by the Hosotani mechanism
}

\section{Oscar Åkerlund}

Institut für Theoretische Physik, ETH Zurich, CH-8093 Zürich, Switzerland

E-mail: oscara@phys.ethz.ch

\section{Philippe de Forcrand*}

Institut für Theoretische Physik, ETH Zurich, CH-8093 Zürich, Switzerland CERN, Physics Department, TH Unit, CH-1211 Geneva 23, Switzerland

E-mail: forcrandephys.ethz.ch

\begin{abstract}
The Hosotani mechanism claims to achieve gauge-symmetry breaking, for instance $S U(3) \rightarrow$ $S U(2) \times U(1)$. To verify this claim, we propose to monitor the stability of a topological defect stable under a gauge subgroup but not under the whole gauge group, like a $U(1)$ flux state or monopole in the case above. We use gauge invariant operators to probe the presence of the topological defect to avoid any ambiguity introduced by gauge fixing. Our method also applies to an ordinary gauge-Higgs system.
\end{abstract}

The 32nd International Symposium on Lattice Field Theory,

23-28 June, 2014

Columbia University New York, $N Y$

\footnotetext{
* Speaker.
} 

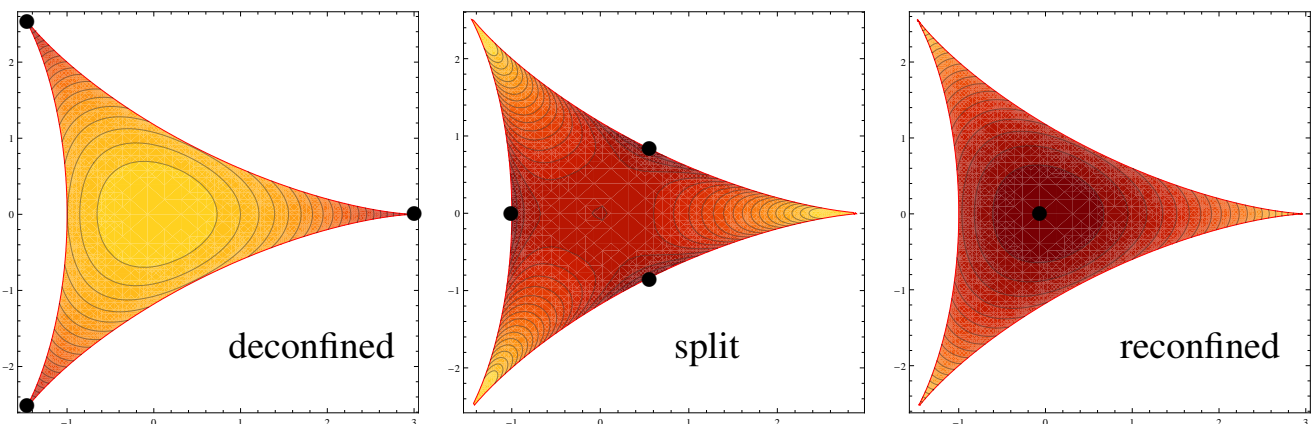

Figure 1: Contour lines of the effective potential $V_{\text {eff }}\left(\operatorname{Tr} P_{5}\right)$ in the three phases, resulting from different matter contents and boundary conditions in the extra dimension. Black dots mark the minima in each case.

\section{Introduction}

Dimensional reduction tells us that QCD at high temperature can be effectively described as a $3 d$ Yang-Mills theory, plus an adjoint Higgs field generated by the static mode of $A_{0}$, i.e. by the Polyakov loop. Dimensional reduction also occurs in the case of a compact extra dimension: a $(4+1) d$ Yang-Mills theory is effectively described as a $4 d$ Yang-Mills, plus an adjoint Higgs field coming from the Polyakov loop $P_{5}$ in the extra dimension. This led Hosotani [1], in 1983, to the scenario of "gauge-Higgs unification": by a judicious choice of matter content and boundary conditions in the extra dimension, the minimum of the effective potential for $\operatorname{Tr} P_{5}$ can be displaced from its trivial value $A_{5}=0$. Then, the corresponding $4 d$ adjoint Higgs field acquires a nontrivial vacuum expectation value, which can [partially] break the gauge symmetry of the YangMills theory. While this scenario seems to be disfavored phenomenologically, we are concerned here with a different aspect: how can one diagnose the claimed breaking of gauge symmetry? Our proposal actually applies also to genuine (gauge + Higgs) systems.

\section{The Hosotani mechanism}

We consider a $(4+1)$-dimensional theory with gauge group $S U(3)$ and a compact extra dimension. The effective potential $V_{\text {eff }}\left(\operatorname{Tr} P_{5}\right)$ can be obtained in perturbation theory, by considering the static modes of the gauge and matter fields. As shown by Hosotani, adjoint fermions with periodic boundary conditions in the extra dimension give a contribution opposite to that of gluons, and can displace the minimum of $V_{\text {eff }}$ from the trivial one (Fig. 1 left) to that of Fig. 1 middle or right. The 3 cases have been dubbed "deconfined", "split" and "reconfined". They exist also in a $(3+1)$-dimensional system, where they were found in a serendipitous way [2], in a project aimed at understanding center symmetry breaking in $\mathrm{QCD}(\mathrm{Adj})$ as proposed by Unsal [3].

Let us consider the diagonalized form of the $S U(3)$ matrix $P_{5}$ in the 3 cases. Up to eigenvalue permutations and global phases $\exp \left( \pm i \frac{2 \pi}{3}\right)$, one finds

$\left(\begin{array}{ccc}+1 & 0 & 0 \\ 0 & +1 & 0 \\ 0 & 0 & +1\end{array}\right)$ ("deconfined"), $\left(\begin{array}{cc|c}-1 & 0 & 0 \\ 0 & -1 & 0 \\ \hline 0 & 0 & +1\end{array}\right)$ ("split"), and $\left(\begin{array}{cc|c}e^{+i \frac{2 \pi}{3}} & 0 & 0 \\ 0 & e^{-i \frac{2 \pi}{3}} & 0 \\ \hline 0 & 0 & 1\end{array}\right)$ ("reconfined").

While the first matrix, the identity, is invariant under any $S U(3)$ gauge transformation $P_{5} \leftarrow \Omega^{\dagger} P_{5} \Omega$ 
$\forall \Omega \in S U(3)$, this is not true of the other two matrices. In the "split" case, $P_{5}$ is left unchanged only if $\Omega \in S U(2) \times U(1)$. In the "reconfined" case, $P_{5}$ is left unchanged only if $\Omega=\exp \left(i \theta_{3} \lambda_{3}+i \theta_{8} \lambda_{8}\right)$, i.e. $\Omega \in U(1) \times U(1)$. Therefore, we are in a situation where the action maintains full $S U(3)$ gauge invariance, but the vacuum does not (in the split and reconfined cases): this characterizes the breaking of the gauge symmetry.

The situation is no different from that of an ordinary Higgs field: one says that the gauge symmetry "breaks" when the Higgs field "develops an expectation value", although the action remains gauge-invariant and the expectation value $\langle\phi\rangle$ remains exactly zero (in the absence of gauge-fixing). Here too, $\left\langle A_{5}\right\rangle$ remains exactly zero under the action of gauge transformations $\Omega$ which permute the eigenvalues of $P_{5}$.

Nevertheless, the long-distance physics of the 3 phases is dramatically different: the $4 d$ theory has 8 gluons in the first case, or 3 gluons and 1 photon in the second case, or 2 photons in the third. The presence of photons gives rise to a Coulomb potential between corresponding probe charges. Yet, these different physics are very hard to recognize, because the corresponding $S U(2)$ and $U(1)$ gauge subgroups are "scrambled" differently at each lattice site.

Let us focus on the "split" $S U(2) \times U(1)$ phase. One might consider detecting the Coulomb potential by measuring $S U(3)$ Wilson loops. But this will not work: each link is a product of an $S U(2)$ and a $U(1)$ element, and the trace of such a loop will obey an area law coming from the $S U(2)$ factors, regardless of the $U(1)$ factors. Clearly, finding a signature of the above phenomena in the infrared properties of the effective $4 d$ theory is not as simple as one might initially think. One way out would perhaps be to fix the gauge ${ }^{1}$, with a gauge condition which minimizes the magnitude of the $S U(3)$ link elements which do not belong to $S U(2) \times U(1)$ or $U(1) \times U(1)$. The latter is the well-known Maximal Abelian gauge. Here, we want to study gauge-invariant observables, and follow a different route.

\section{Gauge symmetry breaking seen in a gauge-invariant way}

Since the eigenvalues of $P_{5}$ are gauge-invariant, it is instructive to consider the quadratic fluctuations $m_{k}^{-2}=\left\langle\left(\overline{A_{5}{ }^{k}}-\left\langle\overline{A_{5}}{ }^{k}\right\rangle\right)^{2}\right\rangle$ of the static mode $\overline{A_{5}}$ defined via $P_{5}=\exp \left(i g L_{5} \overline{A_{5}}{ }^{k} \lambda_{k}\right)$. These fluctuations determine the Higgs mass squared. A possible $k$-dependence will be a clear sign of gauge-symmetry breaking. These masses can be determined by perturbation theory around the vacuum corresponding to each phase. They are shown in Fig. 2 [5]. As one would expect, fluctuations of $\overline{A_{5}}$ about the trivial vacuum in the deconfined phase are isotropic in color space. The same is true in the reconfined phase, where $Z_{3}$ symmetry is enforced in $P_{5}$ locally. Remarkably, in the split phase two different masses are found, for fluctuations of $\overline{A_{5}}$ in the $\lambda_{3}$ and $\lambda_{8}$ directions. In other words, $V_{\text {eff }}\left(\overline{A_{5}}\right)$ is elliptical, not spherical, in the split phase. However, this interesting phenomenon is of no relevance to long-distance $4 d$ physics, because the Higgs mass is of order $1 / L_{5}$.

Our proposal is to monitor the stability of topological excitations supported by a gauge subgroup in the case of gauge-symmetry breaking, but not by the whole gauge group. The simplest example is provided by $\Pi_{1}(U(1))=\mathscr{Z}$, while $\Pi_{1}(S U(3))=\Pi_{1}(S U(2))=\mathbf{1}$. The corresponding topological excitation, an Abelian flux in some plane $x y$, is going to be stable if the gauge symmetry is broken down to a subgroup $U(1)$, but will otherwise unwind in a larger $S U(3)$ or $S U(2)$

\footnotetext{
${ }^{1}$ No signal was found by Jim Hetrick, as presented in [4].
} 


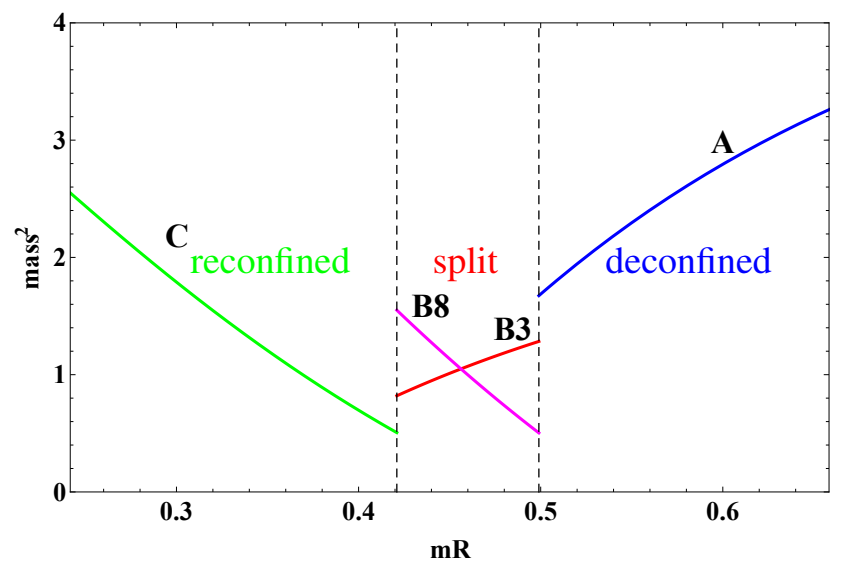

Figure 2: Mass squared of the Higgs field, as a function of the size of the extra dimension, for two adjoint fermions. In the split phase, the two Abelian components of the Higgs field have different masses. Perturbative calculation and figure from [5].

gauge group. The same happens with an Abelian monopole. The stability of these objects can be monitored via gauge-invariant observables like the plaquette.

Let us first consider an $x y$ Abelian flux in a $U(1)$ gauge theory on an $L_{x} \times L_{y} \times L_{z} \times L_{t}$ lattice. To prepare such a state, start from a "cold" configuration $U_{\mu}(x)=\mathbf{1} \forall \mathbf{x}, \mu$. Then, in each $x y$ plane, insert a $2 \pi$ flux by arranging the links so that each $x y$ plaquette $P_{x y}$ is equal to $\exp \left(i \frac{2 \pi}{L_{x} L_{y}}\right)$. From this starting configuration, perform usual Monte Carlo updates, and monitor the gauge-invariant flux action: $\Delta=\left\langle\operatorname{Tr} U_{P_{x z}}\right\rangle-\left\langle\operatorname{Tr} U_{P_{x y}}\right\rangle$, where the difference is taken to isolate the effect of the $x y$ flux. Classically, $\Delta=1-\cos \frac{2 \pi}{L_{x} L_{y}} \approx \frac{2 \pi^{2}}{L_{x}^{2} L_{y}^{2}}$. The leading effect of fluctuations is to modify $P_{x y}$ and $P_{x z}$ in the same way, so that $\Delta \sim\left\langle\operatorname{Tr} U_{P_{x z}}\right\rangle\left(1-\cos \frac{2 \pi}{L_{x} L_{y}} B\right)$ for $B$ units of flux. This simple prediction is completely consistent with the numerical simulation of Fig. 3, where $B$ is incremented every 50 Monte Carlo sweeps. Flux states are extremely stable, since for their decay one $x y$ plaquette in each plane must go through angle $\pi$. This only happens at the right edge of the figure.

We can now repeat this construction in the case of $S U(3)$ gauge-symmetry breaking. Starting from a "cold" 2 configuration, prepare a flux state in some specific $U(1)$ subgroup, in each $x y$ plane: $\theta_{U(1)}=\frac{2 \pi}{L_{x} L_{y}}$. Then perform ordinary Monte Carlo sweeps of the $S U(3)$ link variables, but with an action which is expected to induce gauge-symmetry breaking. In our case, we chose to induce gauge-symmetry breaking by applying an external potential $h_{F} \operatorname{Re} \operatorname{Tr} P_{5}+h_{A}\left|\operatorname{Tr} P_{5}\right|^{2}$, following [6]. This is simpler and computationally much cheaper than simulating adjoint fermions.

One effect of the $S U$ (3) Monte Carlo updates is to rotate in $S U(3)$ the $U(1)$ subgroup where the flux was introduced, independently at each lattice site. To some extent, this local scrambling could be undone by gauge-fixing. But gauge-fixing is not necessary: what we want to ascertain is whether the $U(1)$ flux is still there. For that purpose, it is sufficient to monitor the gauge-invariant excess action in the $x y$ planes, namely $\Delta=\left\langle\operatorname{Tr} U_{P_{x z}}\right\rangle-\left\langle\operatorname{Tr} U_{P_{x y}}\right\rangle$, just like in the pure $U(1)$ case.

Fig. 4 shows the results of such an experiment in the reconfined phase $(S U(3) \rightarrow U(1) \times U(1))$. On the $y$-axis, $\Delta$ has been normalized to its value in the pure $U(1)$ case. Interestingly, the excess

\footnotetext{
2"Cold" here stands for a state which minimizes the total lattice action, with non-trivial $P_{5}$.
} 


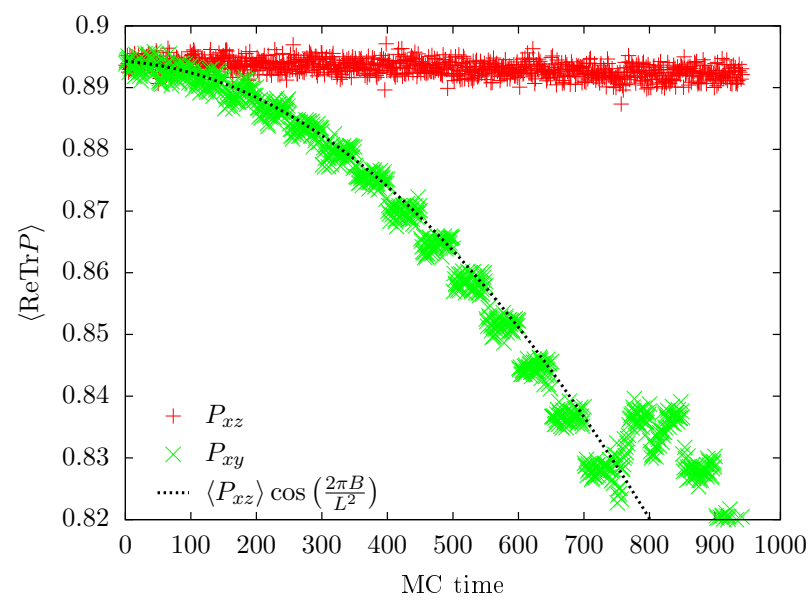

Figure 3: Average $x y$ and $x z$ plaquettes in a $U(1)$ system, under the introduction of one unit of $x y$ magnetic flux every 50 sweeps. The dotted line is the leading prediction. Flux states are long-lived and cause a shift of the in-plane plaquette.

action depends on the $U(1)$ subgroup where the flux is introduced. The reason is the following. In all cases, a $U(1)$ angle $\theta=\frac{2 \pi}{L_{x} L_{y}}$ is introduced in each $x y$ plaquette. The corresponding action is $1-\operatorname{Tr}\left(e^{i \theta}\right) \approx \frac{1}{2} \theta^{2}$ in the pure $U(1)$ system. But in the $S U(3)$ system, if the flux is introduced in the $\lambda_{3}$ subgroup, the corresponding action is $1-\frac{1}{3} \operatorname{Tr}\left\{\operatorname{diag}\left(e^{i \theta}, e^{-i \theta}, 1\right)\right\} \approx \frac{1}{3} \theta^{2}$. And if the flux is introduced in the $\lambda_{8}$ subgroup, the action is $1-\frac{1}{3} \operatorname{Tr}\left\{\operatorname{diag}\left(e^{i \theta}, e^{i \theta}, e^{-2 i \theta}\right)\right\} \approx \theta^{2}$. Thus, a magnetic flux in the $\lambda_{3}$ or $\lambda_{8}$ subgroup incurs an action equal to $2 / 3$ or 2 times that in a $U(1)$ system, respectively. This is precisely what Fig. 4 shows, with horizontal dotted lines corresponding to $2 / 3$ (subgroup $\lambda_{3}$ ), 2 (subgroup $\lambda_{8}$ ) or $14 / 3$ ( 2 units of flux in $\lambda_{3}$ plus 1 unit in $\lambda_{8}$ ). Note that these topological excitations are extremely stable: their sudden decay after 1000 Monte Carlo sweeps is due to our turning off the external potential which maintained the reconfined phase. Then, the full $S U$ (3) gauge symmetry is immediately restored, and the $U(1)$ fluxes can freely unwind.

The next topological defect we have considered is a magnetic monopole, which is visible via its gauge-invariant $3 d$ magnetic flux through the 6 faces of an elementary cube (see Fig. 5 left). Actually, we are interested in a classical monopole, obtained by minimizing the action of a $3 d$ $U(1)$ lattice of size $L^{3}$ containing one monopole. To enforce the presence of one monopole, we introduce a flux $\pi$ through 3 of the faces of the lattice, and choose charge-conjugated periodic boundary conditions in each of the 3 directions, $U_{\mu}(x+L)=U_{\mu}^{*}(x)$. The resulting construction, Fig. 5 right, is the analogue of the DeGrand-Toussaint monopole on the left, but on the scale $L$ instead of $a$. It was already used in [7] to measure the monopole mass, but the numerical results presented there turn out to be incorrect.

Fig. 6 left shows the minimum energy (measured by cooling) of a $U(1)$ magnetic monopole induced by the above boundary conditions, as a function of the size $L$ of the cubic lattice. To understand its $L$-dependence, consider first a monopole of charge $Q_{M}=2 \pi / e$ in the continuum. The energy of the magnetic field inside a sphere of radius $R$ is $E(R)=2 \pi \int_{0}^{R} d r r^{2} B(r)^{2}$. It is UV-divergent, and the lattice spacing $a$ will cutoff the integral and regularize the divergence. In the infrared, since $B(r)=Q_{M} /\left(4 \pi r^{2}\right)$ as dictated by Gauss' law, one obtains $E(R=\infty)-E(R)=$ 


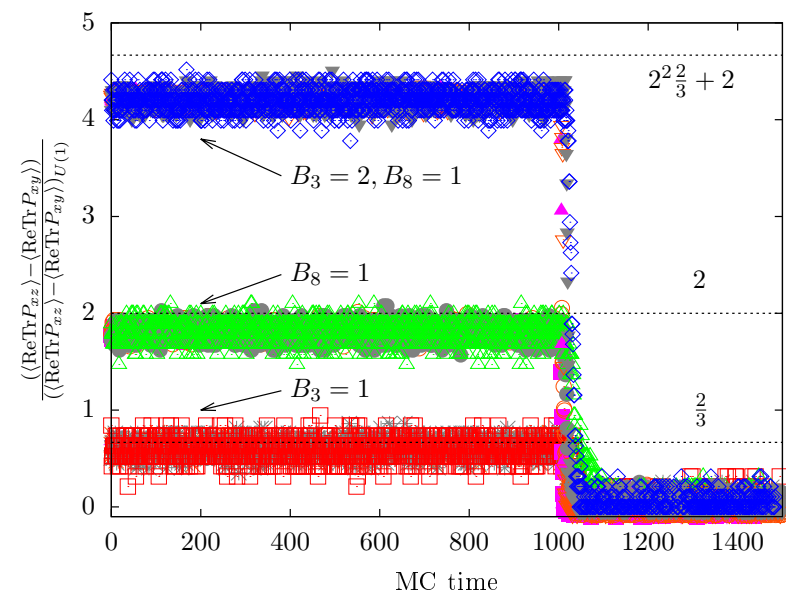

Figure 4: In an $S U(3)$ system in the reconfined phase $(U(1) \times U(1))$, magnetic flux is introduced in the $x y$ plane. The corresponding shift in the $x y$ plaquette, normalized to that in a pure $U(1)$ system, is shown for several flux combinations in the $\lambda_{3}$ and $\lambda_{8}$ subgroups. The dotted lines show the classical predictions. After 1000 sweeps, the gauge-symmetry breaking potential is turned off: the full gauge-symmetry is restored and the flux states decay immediately.

$\frac{Q_{M}^{2}}{8 \pi} \int_{R}^{\infty} d r 1 / r^{2}=\frac{1}{e^{2}} \frac{\pi}{2 R}$. This calculation is slightly modified in a cubic box with $C$-periodic boundary conditions. The boundary conditions generate an infinite array of mirror charges, arranged in a cubic array of spacing $L$ and alternating in sign, as in an $\mathrm{Na}^{+} \mathrm{Cl}^{-}$crystal. They interact via a $1 / r$ potential, so that the energy of the array is proportional to $\alpha_{3}=\sum_{i j k}^{\prime} \frac{(-1)^{i+j+k}}{\sqrt{i^{2}+j^{2}+k^{2}}}=-1.74756 .$. , which is called Madelung's constant. The resulting monopole energy correction is $E(R=\infty)-E(R)=$ $\frac{1}{e^{2}} \frac{\alpha_{3}}{2} \frac{\pi}{L}=\frac{2.745 . .}{L}$. This is precisely the $1 / L$ dependence seen in Fig. 6 left. Since its origin is infrared, this term is universal, i.e. independent of the lattice action considered. The leading term of course depends on the form of the ultraviolet cutoff, and thus is action-dependent. The additional, $1 / L^{3}$, tiny corrections come from $(a / L)^{2}$ lattice corrections to the continuum Coulomb potential.

Now, as in the case of $U(1)$ fluxes, we can introduce a $U(1)$ monopole in a subgroup of an $S U$ (3) configuration. If the gauge symmetry is broken by the external Polyakov loop potential, the $U(1)$ monopole is stable, and we can measure its energy by cooling. The results are shown Fig. 6 right. First, the monopole energy depends on the $U(1)$ subgroup chosen, just like for fluxes. This fact was first noticed in Ref. [8] which used Maximal Abelian gauge and Abelian projection to isolate the monopoles. Here, we obtain precise values for the monopole energies in the thermodynamic limit: contrary to the energies of flux states, the monopole energies are not obtained by applying a simple factor to the $U(1)$ case, because the UV-regularization of the monopole field differs in the different subgroups. Nevertheless, the coefficient of the $1 / L$ correction, which comes from IR effects, varies as for flux states: it is $2 / 3$ and 2 times the $U(1)$ value for subgroups spanned by $\lambda_{3}$ and $\lambda_{8}$, respectively. Moreover, the $1 / L^{3}$ coefficients scale in the same way, since they are all caused by the same lattice distortion of the Coulomb potential.

The topological excitations considered here, Abelian fluxes and monopoles, are appropriate for diagnosing gauge-symmetry breaking to a $U(1)$ subgroup. To diagnose gauge-symmetry breaking to an $S U(2)$ subgroup, one should monitor the stability of a 't Hooft-Polyakov monopole. 

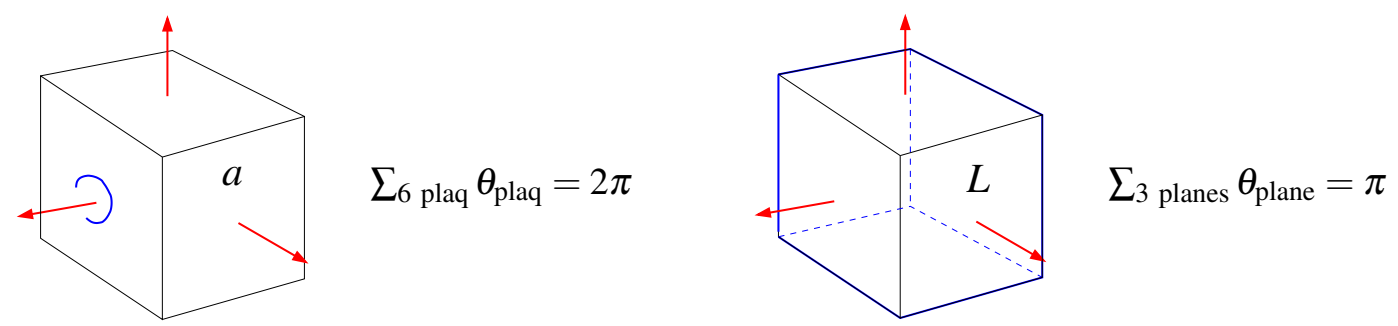

Figure 5: (left) DeGrand-Toussaint magnetic monopole in an elementary cube of size $a$. (right) The same construction on the scale $L$ of the whole lattice ensures the presence of a magnetic monopole somewhere inside. Charge-conjugated boundary conditions are required to obtain non-zero fluxes at the boundary.

$\mathrm{U}(1)$

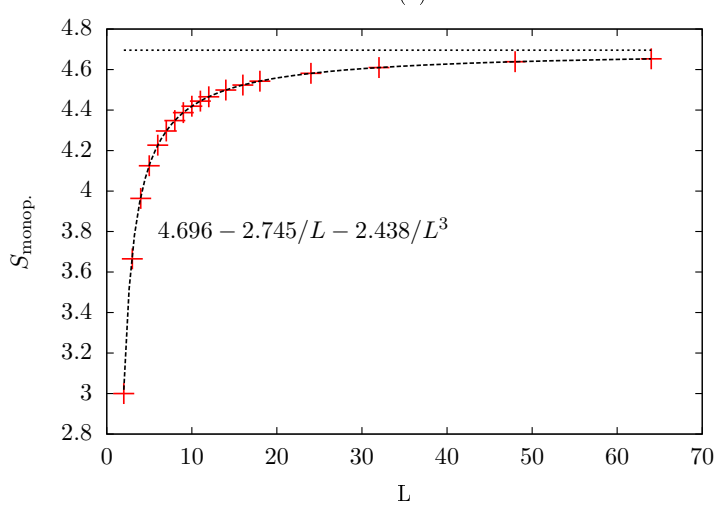

$\mathrm{U}(1) \subset \mathrm{SU}(3)$

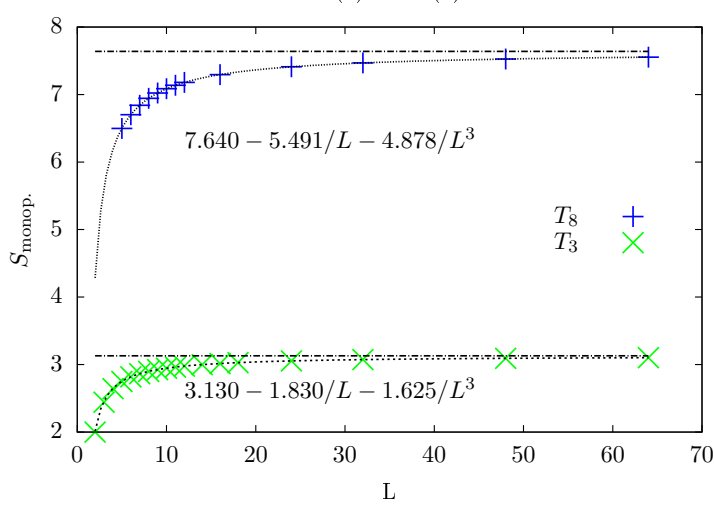

Figure 6: (left) Minimum action of a $U(1)$ magnetic monopole as a function of the size $L$ of the cubic lattice. The $1 / L$ correction is caused by the cubic array of image-charges with alternating signs, and its magnitude is exactly given by Madelung's constant. (right) Same, for a $U(1)$ magnetic monopole in the $\lambda_{3}$ or $\lambda_{8}$ sector of an $S U(3)$ system in the reconfined $(U(1) \times U(1))$ phase. The two types of monopoles have different masses. The $1 / L$ correction is given by the correspondingly rescaled Madelung constant.

This next step is under investigation. Finally, it is clear that our approach can be used without change to diagnose gauge-symmetry breaking in an ordinary gauge-Higgs system. Note that our construction is completely non-local, so that it does not contradict the Fradkin-Shenker argument against the existence of an order parameter distinguishing the Higgs and the confining regimes.

\section{References}

[1] Y. Hosotani, Phys. Lett. B 126 (1983) 309. Phys. Lett. B 129 (1983) 193.

[2] G. Cossu and M. D’Elia, JHEP 0907 (2009) 048.

[3] M. Unsal and L. G. Yaffe, Phys. Rev. D 74 (2006) 105019 [hep-th/0608180]; P. Kovtun, M. Unsal and L. G. Yaffe, JHEP 0706 (2007) 019.

[4] J. E. Hetrick, PoS LATTICE 2013, 102 (2014).

[5] G. Cossu, H. Hatanaka, Y. Hosotani and J. I. Noaki, Phys. Rev. D 89, no. 9, 094509 (2014).

[6] J. C. Myers and M. C. Ogilvie, Phys. Rev. D 77 (2008) 125030.

[7] M. Vettorazzo and P. de Forcrand, Nucl. Phys. B 686, 85 (2004) [hep-lat/0311006].

[8] P. Cea and L. Cosmai, Phys. Rev. D 62, 094510 (2000) [hep-lat/0006007]. 